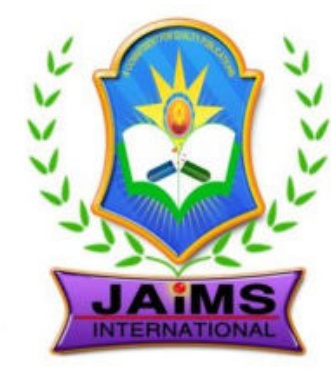

ISSN 2456-3110

Vol $5 \cdot$ Issue 1

Jan-Feb 2020

Journal of

Ayurveda and Integrated Medical Sciences

www.jaims.in

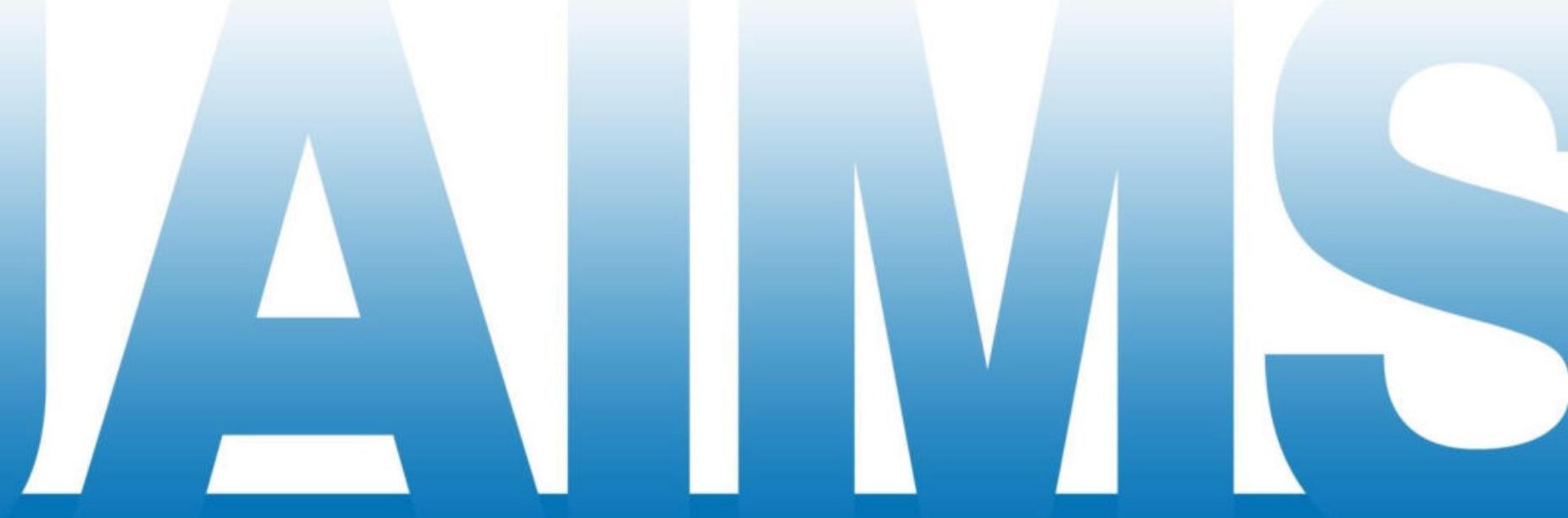

An International Journal for Researches in Ayurveda and Allied Sciences

\title{
Charaka
}




\title{
Ayurveda and Integrated Medical Sciences
}

\section{Clinical efficacy of Panchatikta Kshira Basti in the management of Gridhrasi}

\author{
Dr. Mridula Pathak', Dr. Ashish Mhatre ${ }^{2}$
}

${ }^{1}$ Post Graduate Scholar, ${ }^{2}$ Associate Professor, Dept. of Kayachikitsa, D. Y. Patil School of Ayurveda, Nerul, Navi Mumbai, Maharashtra, INDIA.

\section{A B S TR A C T}

\begin{abstract}
The study was undertaken to evaluate the clinical efficacy of Panchatikta Kshira Basti in the management of Sciatica. Panchatikta Kshira Basti contains Guduchi (Tinospora cardiofolia). Nimba (Azadirakta indica) Vasa (Adhathoda vasica), Kantakari (Solanum Surrattense), Patola (Trichosanthes dioica), Kshira (Milk) Goghrita (Ghee), Madhu (Honey), Saindhava (Salt). This remedy prove extremely beneficial as it can be performed on the OPD and IPD basis, gives significant relief in the symptoms e.g. radiating pain, stiffness, twitching sensation. The subjective parameters like Pain, SLR, VAD, VDS etc., were used to score clinical outcome. The average clinical improvement was calculated by proper statistical treatment. Our experience with this modality has been encouraging as the response pattern is good in considerably short duration of treatment. The patient improves gradually after 4 weeks of treatment. The sustained improvement with Basti presents a window of opportunity in the clinical management of Sciatica. Ideally this technique should be practically taught to the physicians and should be evaluated scientifically using principles of biophysics and nerve conduction studies.
\end{abstract}

Key words: Sciatica, Ghridhrasi, Panchatikta Kshira Basti.

\section{INTRODUCTION}

Sciatica is characterized by radiating pain in an area of the leg typically served by one nerve root in the lumbar or sacral spine. The most common cause of sciatica includes herniated disc, degenerative disc disease and lumbar spinal stenosis. The estimated annual incidence of sciatica in western countries is 5 cases per 1000 adults. Lumbar spine disorders rank $5^{\text {th }}$ among disease categories in the cost of hospital care and account for highest costs resulting from

\section{Address for correspondence:}

\section{Dr. Mridula Pathak}

Post Graduate Scholar, Dept. of Kayachikitsa, D. Y. Patil School of

Ayurveda, Nerul, Navi Mumbai, Maharashtra, INDIA.

E-mail: pmridula27@gmail.com

Submission Date: 16/01/2020 Accepted Date: 23/02/2020

\begin{tabular}{|l|l|} 
Submission Date: 16/01/2020 Accepted Date: 23/02/2020 \\
\hline Quick Response Code & Website: www.jaims.in \\
\hline DOI: 10.21760/jaims.5.1.9
\end{tabular}

absenteeism from work and disability than any other category.

About $2 \%$ to $40 \%$ of people have sciatica. This incidence is related to age, rare before 20 years. Sciatica has been reported to occur in 1 to $10 \%$ of the population, most commonly in people age 25 to 45 years. Men are more frequently affected than women. The highest incidence is found in the fifth decade and then decreases with increasing age. Regular walking also was found to increase the incidence of sciatica.

\section{AIMS AND OBJeCTIVES}

To clinically evaluate the efficacy of Panchatikta Kshira Basti on the basis of scientific Ayurvedic principles in reversing or halting the process of Sciatica.

\section{Materials And Methods}

\section{Source of Materials}

Raw materials were collected from the department of Rasashastra and Bhaishajya Kalpana, Dr. D.Y. Patil 
College of Ayurved and Hospital and Research Institute, Nerul, Navi Mumbai, and medicines were prepared classically in the Pharmacy of Rasashastra and Bhaishajya Kalpana.

\section{Research Place}

1. A clinical survey of subjects attending OPD and IPD of Department of Kaya Chikitsa, Dr. D.Y. Patil College of Ayurveda and Hospital and Research Institute, Nerul, Navi Mumbai were included and subjects fulfilling the criteria of diagnosis as per the Performa have been selected for the study.

2. The data, which was obtained by the clinical trial was statistically analyzed by applying Student ${ }^{\prime} t{ }^{\prime}$ test.

\section{Inclusion Criteria}

1. Irrespective of age, sex, race and religion

2. Patient's with positive S.L.R Test

3. Radiologically Confirmed Disc Herniation

4. Lumbo-sacral radicular syndrome lasted for 6-12 weeks

\section{Exclusion Criteria}

Patients with the following diseases or Surgeries

1. Metabolic disorders like Diabetes Mellitus etc.

2. Skin diseases.

3. Infectious disease e.g. HIV positive, HbsAg positive, Koch's

4. Pott's disease, cauda equina syndrome, Muscle paralysis

5. Previous spine surgeries, Bony stenosis Spondylolisthesis,

6. Pregnancy.

\section{Methodology Used}

\begin{tabular}{|l|l|}
\hline $\begin{array}{l}\text { Details of } \\
\text { Procedure }\end{array}$ & Panchatikta Kshira Basti \\
\hline $\begin{array}{l}\text { No. of } \\
\text { Patient }\end{array}$ & 30 \\
\hline
\end{tabular}

\begin{tabular}{|c|c|c|}
\hline Ingredients & $\begin{array}{l}\text { Guduchi } \\
\text { Nimba Patra } \\
\text { VasaPatra } \\
\text { Kantakari Churna } \\
\text { Patola Churna } \\
\text { Milk } \\
\text { Ghrita } \\
\text { Honey } \\
\text { Saindhava }\end{array}$ & $\begin{array}{l}-10 \mathrm{gm} \\
-10 \mathrm{gm} \\
-10 \mathrm{gm} \\
-10 \mathrm{gm} \\
-10 \mathrm{gm} \\
-200 \mathrm{ml} \\
-40 \mathrm{ml} \\
-20 \mathrm{ml} \\
-5 \mathrm{gm}\end{array}$ \\
\hline $\begin{array}{l}\text { Vidhi } \\
\text { (Procedure) } \\
\text { a) Purva } \\
\text { Karma }\end{array}$ & \multicolumn{2}{|l|}{$\begin{array}{l}\text { 1) Abhyanga } \\
\text { 2) Swedan } \\
\text { 3) Bhojana } \\
\text { 4) Chankraman }\end{array}$} \\
\hline $\begin{array}{l}\text { b) Pradhana } \\
\text { Karma }\end{array}$ & \multicolumn{2}{|c|}{$\begin{array}{l}\text { 1) Vam Parshwa Avastha Shayan } \\
\text { 2) Bastipranidhan } \\
\text { 3) Sphika Tadana }\end{array}$} \\
\hline $\begin{array}{l}\text { c) Paschat } \\
\text { Karma }\end{array}$ & \multicolumn{2}{|c|}{$\begin{array}{l}\text { 1) Bastipratygaman and observation of Yog- } \\
\text { Atiyog Lakshana } \\
\text { 2) Pathyapathya } \\
\text { 3) Observation of Sneha Vyapada if any and their } \\
\text { treatment }\end{array}$} \\
\hline Dose & \multicolumn{2}{|l|}{$260 \mathrm{ml} /$ day } \\
\hline Kala & \multicolumn{2}{|c|}{ Prataha (Abhakata) } \\
\hline Type of Basti & \multicolumn{2}{|l|}{ Niruhavata } \\
\hline $\begin{array}{l}\text { Route of } \\
\text { Administrati } \\
\text { on }\end{array}$ & \multicolumn{2}{|l|}{ per Rectal } \\
\hline Duration & \multicolumn{2}{|l|}{16 Days } \\
\hline
\end{tabular}

\section{Drug Specifications}

\section{Contents of Panchatikta Kshira Basti}

\begin{tabular}{|l|l|l|l|l|l|l|}
\hline $\begin{array}{l}\text { S } \\
\text { N }\end{array}$ & $\begin{array}{l}\text { Name } \\
\text { of Drug }\end{array}$ & Rasa & Virya & $\begin{array}{l}\text { Vipak } \\
\mathbf{a}\end{array}$ & Guna & $\begin{array}{l}\text { Doshaghna } \\
\text { ta }\end{array}$ \\
\hline 1. & $\begin{array}{l}\text { Guduch } \\
\text { i }\end{array}$ & $\begin{array}{l}\text { Tikta, } \\
\text { Kashay } \\
\text { a }\end{array}$ & $\begin{array}{l}\text { Ushn } \\
\text { a }\end{array}$ & $\begin{array}{l}\text { Madh } \\
\text { ur }\end{array}$ & $\begin{array}{l}\text { Guru, } \\
\text { Snigdh } \\
\text { a }\end{array}$ & Tridosha \\
\hline 2. & Nimba & $\begin{array}{l}\text { Tikta, } \\
\text { Kashay } \\
\text { a }\end{array}$ & Shita & Katu & $\begin{array}{l}\text { Laghu, } \\
\text { Ruksh } \\
\text { a }\end{array}$ & $\begin{array}{l}\text { Kapha, } \\
\text { Pitta }\end{array}$ \\
\hline
\end{tabular}




\begin{tabular}{|l|l|l|l|l|l|l|}
\hline 3. & Vasa & $\begin{array}{l}\text { Tikta, } \\
\text { Kashay } \\
\text { a }\end{array}$ & Shita & Katu & $\begin{array}{l}\text { Laghu, } \\
\text { Ruksh } \\
\text { a }\end{array}$ & $\begin{array}{l}\text { Kapha, } \\
\text { Pitta }\end{array}$ \\
\hline 4. & $\begin{array}{l}\text { Kantaka } \\
\text { ri }\end{array}$ & $\begin{array}{l}\text { Tikta, } \\
\text { Katu }\end{array}$ & $\begin{array}{l}\text { Ushn } \\
\text { a }\end{array}$ & Katu & $\begin{array}{l}\text { Laghu, } \\
\text { Ruksh } \\
\text { a }\end{array}$ & $\begin{array}{l}\text { Kapha, } \\
\text { Pitta }\end{array}$ \\
\hline 5. & Patola & Tikta & $\begin{array}{l}\text { Ushn } \\
\text { a }\end{array}$ & Katu & $\begin{array}{l}\text { Laghu, } \\
\text { Ruksh } \\
\text { a }\end{array}$ & Tridosha \\
\hline
\end{tabular}

Parameter of Assessment

Clinical assessment was done under these basic subjects;

\section{1) Assessment of Efficacy}

A) Subjective improvement

B) Objective improvement

2) Assessment of Tolerability and other procedure

e.g. - premature discontinuation

- Incomplete case

- Unsatisfactory or cured case

\section{1) Assessment of Efficacy}

Patients were weekly assessed under these guidelines:-

\section{A) Subjective Assessment}

Assessment of signs and symptoms were done in every week for 4 weeks. The specific criteria adopted for gradation of pain and tenderness. To assess the improvement in clinical symptoms of Sciatica patients were arbitrarily graded into four grades ( 0 to 3 ) on the basis of severity-duration-stage.

Study Design $\rightarrow$ Screening of Subject for Inclusion (Counselling, Informed and Consent Taken) $\rightarrow$ Single Arm Clinical Study $\rightarrow$ Panchatikta Kshira Basti $\rightarrow$ Initial Assessment (Dietary and Behavioral Advice) $\rightarrow$ Intervention $\rightarrow$ Assessment at the End of Treatment $\rightarrow$ Final Assessments at the End of 4 Weeks (For Evidence of Relapse of Signs and Symptoms, if any) $\rightarrow$ Statistical Analysis.

\section{Method of preparation - Panchatikta Kshira.}

- Panchatikta-Siddha-Kshira was prepared by Kshirpaka method as mentioned in Sharangadhara Samhita with the help of Madhyama Agni.

- The Panchatikta dravyas were smashed and Kalka was made.

- According to Kwath Kalpana [Kalka: water 1:16] $800 \mathrm{ml}$ water was added and boiled until $200 \mathrm{ml}$ was remained, kwatha [decoction] was filtered and $200 \mathrm{ml}$ of milk was added. This mixture was again boiled on dim fire until $200 \mathrm{ml}$ remained.

- In this $40 \mathrm{ml}$ ghee, $20 \mathrm{ml}$ honey and $5 \mathrm{gm}$ Saindhava were added and mixed properly. This $260 \mathrm{ml}$ Basti dravya was used for Basti.

Gradation of Clinical Symptoms

\begin{tabular}{|l|l|}
\hline Clinical Symptom & Pain \\
\hline Grade 0 & No pain \\
\hline Grade 1 & $\begin{array}{l}\text { Mild (+) pain - Complained by patient when } \\
\text { asked }\end{array}$ \\
\hline Grade 2 & $\begin{array}{l}\text { Moderate (++) - Patient frequently complains } \\
\text { of pain and has painful look }\end{array}$ \\
\hline Grade 3 & $\begin{array}{l}\text { Severe (+++) - Excruciating pain associated } \\
\text { with painful cries and agonizing look }\end{array}$ \\
\hline
\end{tabular}

\begin{tabular}{|c|c|}
\hline $\begin{array}{l}\text { Clinical } \\
\text { Symptoms }\end{array}$ & Tenderness \\
\hline Grade 0 & No - Tenderness \\
\hline Grade 1 & $\begin{array}{l}\text { Mild }(+) \text { - Patient Winces after digital } \\
\text { pressure }\end{array}$ \\
\hline Grade 2 & $\begin{array}{l}\text { Moderate }(++)-\text { Patient winces and } \\
\text { withdrawals' the affected part }\end{array}$ \\
\hline Grade 3 & $\begin{array}{l}\text { Severe }(+++) \text { - Patient doesn't allow to touch } \\
\text { the affected part }\end{array}$ \\
\hline
\end{tabular}


Gradation of Clinical Signs

\begin{tabular}{|l|l|l|}
\hline Clinical & Signs & SLR Test \\
\hline Absent & 0 & SLR $90^{\circ}$ \\
\hline Mild & $1(+)$ & SLR $61-80^{\circ}$ \\
\hline Moderate & $2(++)$ & SLR $31-60^{\circ}$ \\
\hline Severe & $3(+++)$ & SLR 0-30 \\
\hline
\end{tabular}

\begin{tabular}{|l|l|l|}
\hline Clinical & Signs & Lasegue's sign \\
\hline Absent & 0 & No pain \\
\hline Mild & $1(+)$ & $\begin{array}{l}\text { Mild increase in pain on SLR, following } \\
\text { foot dorsiflexion }\end{array}$ \\
\hline Moderate & $2(++)$ & $\begin{array}{l}\text { Moderate increase in pain on SLR, } \\
\text { following foot dorsiflexion }\end{array}$ \\
\hline Severe & $3(+++)$ & $\begin{array}{l}\text { Severe increase in pain on SLR, } \\
\text { following foot dorsiflexion }\end{array}$ \\
\hline
\end{tabular}

\begin{tabular}{|l|l|l|}
\hline Clinical & Signs & Verbal Dating Scale \\
\hline Absent & 0 & No pain \\
\hline Mild & $1(+)$ & Mild pain \\
\hline Moderate & $2(++)$ & Moderate pain \\
\hline Severe & $3(+++)$ & Worst possible pain \\
\hline
\end{tabular}

\begin{tabular}{|l|l|l|}
\hline Clinical & Signs & Visual Analogue scale \\
\hline Absent & 0 & No pain \\
\hline Mild & $1(+)$ & $0-5$ scale \\
\hline Moderate & $2(++)$ & 05-Oct \\
\hline Severe & $3(+++)$ & Oct-15 \\
\hline
\end{tabular}




\section{ObSERVATIONS AND RESULtS}

- Effect of Panchatikta Kshira Basti in Signs and Symptoms of Sciatica

\begin{tabular}{|l|l|l|l|l|l|l|l|l|}
\hline $\begin{array}{l}\text { Cardin } \\
\text { al }\end{array}$ & \multicolumn{2}{|l|}{$\begin{array}{l}\text { Mean } \\
\text { Score }\end{array}$} & $\begin{array}{l}\text { Me } \\
\text { an }\end{array}$ & SD \pm & S E \pm & 't' & P & $\%$ \\
\hline $\begin{array}{l}\text { Sympt } \\
\text { oms }\end{array}$ & B.T & A.T & & & & & & \\
\hline Pain & 2.1 & 0.2 & 1.9 & 0.6 & 0.14 & $\begin{array}{l}13 . \\
26\end{array}$ & $\begin{array}{l}<0.000 \\
1\end{array}$ & $\begin{array}{l}88 . \\
37\end{array}$ \\
\hline $\begin{array}{l}\text { Tender } \\
\text { ness }\end{array}$ & 1.8 & $\begin{array}{l}0.9 \\
5\end{array}$ & $\begin{array}{l}0.8 \\
5\end{array}$ & $\begin{array}{l}0.3 \\
66\end{array}$ & $\begin{array}{l}0.08 \\
19\end{array}$ & $\begin{array}{l}10 . \\
37\end{array}$ & $\begin{array}{l}<0.000 \\
1\end{array}$ & $\begin{array}{l}47 . \\
22\end{array}$ \\
\hline
\end{tabular}

\begin{tabular}{|c|c|c|c|c|c|c|c|c|}
\hline $\begin{array}{l}\text { Cardin } \\
\text { al }\end{array}$ & $\begin{array}{l}\text { Mea } \\
\text { Scor }\end{array}$ & & $\begin{array}{l}\text { Me } \\
\text { an }\end{array}$ & $S D \pm$ & SE \pm & ' $t$ ' & $\mathbf{P}$ & $\%$ \\
\hline Signs & B.T & A.T & & & & & & \\
\hline $\begin{array}{l}\text { SLR } \\
\text { Test }\end{array}$ & 1.8 & $\begin{array}{l}0.9 \\
5\end{array}$ & $\begin{array}{l}0.8 \\
5\end{array}$ & $\begin{array}{l}0.3 \\
66\end{array}$ & $\begin{array}{l}0.08 \\
19\end{array}$ & $\begin{array}{l}10.3 \\
76\end{array}$ & $\begin{array}{l}<0.00 \\
01\end{array}$ & $\begin{array}{l}47 . \\
22\end{array}$ \\
\hline $\begin{array}{l}\text { Bragar } \\
\text { d's } \\
\text { sign }\end{array}$ & 1.8 & $\begin{array}{l}0.9 \\
5\end{array}$ & $\begin{array}{l}0.8 \\
5\end{array}$ & $\begin{array}{l}0.3 \\
66\end{array}$ & $\begin{array}{l}0.08 \\
19\end{array}$ & $\begin{array}{l}10.3 \\
76\end{array}$ & $\begin{array}{l}<0.00 \\
01\end{array}$ & $\begin{array}{l}47 . \\
22\end{array}$ \\
\hline $\begin{array}{l}\text { Lasegu } \\
\text { e's } \\
\text { sign }\end{array}$ & 1.8 & $\begin{array}{l}0.9 \\
5\end{array}$ & $\begin{array}{l}0.8 \\
5\end{array}$ & $\begin{array}{l}0.3 \\
66\end{array}$ & $\begin{array}{l}0.08 \\
19\end{array}$ & $\begin{array}{l}10.3 \\
76\end{array}$ & $\begin{array}{l}<0.00 \\
01\end{array}$ & $\begin{array}{l}47 . \\
22\end{array}$ \\
\hline $\begin{array}{l}\text { Verbal } \\
\text { Dating } \\
\text { Scale }\end{array}$ & $\begin{array}{l}2.1 \\
5\end{array}$ & $\begin{array}{l}0.2 \\
5\end{array}$ & 1.9 & $\begin{array}{l}0.6 \\
40\end{array}$ & $\begin{array}{l}0.14 \\
3\end{array}$ & $\begin{array}{l}13.2 \\
62\end{array}$ & $\begin{array}{l}<0.00 \\
01\end{array}$ & $\begin{array}{l}88 . \\
37\end{array}$ \\
\hline $\begin{array}{l}\text { Visual } \\
\text { Analog } \\
\text { ue } \\
\text { Scale } \\
\text { (VAS) }\end{array}$ & $\begin{array}{l}2.1 \\
5\end{array}$ & $\begin{array}{l}0.2 \\
5\end{array}$ & 1.9 & $\begin{array}{l}0.6 \\
40\end{array}$ & $\begin{array}{l}0.14 \\
3\end{array}$ & $\begin{array}{l}13.2 \\
62\end{array}$ & $\begin{array}{l}<0.00 \\
01\end{array}$ & $\begin{array}{l}88 . \\
37\end{array}$ \\
\hline
\end{tabular}

\section{- Effect of Panchatikta Kshira Basti in} Investigations in Patients of Sciatica

\begin{tabular}{|c|c|c|c|c|c|c|c|}
\hline \multirow{2}{*}{$\begin{array}{l}\text { Investigati } \\
\text { ons }\end{array}$} & \multicolumn{2}{|c|}{ Mean Score } & \multirow[t]{2}{*}{ SD \pm} & \multirow[t]{2}{*}{ S E \pm} & \multirow[t]{2}{*}{$' \mathrm{t}$ ' } & \multirow[t]{2}{*}{$\mathbf{P}$} & \multirow[t]{2}{*}{$\%$} \\
\hline & B.T & A.T & & & & & \\
\hline \multicolumn{8}{|l|}{$\mathrm{CBC}$} \\
\hline $\mathrm{Hb}$ & $\begin{array}{l}13.1 \\
6\end{array}$ & $\begin{array}{l}13.1 \\
7\end{array}$ & $\begin{array}{l}1.19 \\
3\end{array}$ & $\begin{array}{l}0.26 \\
67\end{array}$ & $\begin{array}{l}0.01 \\
68\end{array}$ & $\begin{array}{l}0.98 \\
67\end{array}$ & $\begin{array}{l}- \\
0.07 \\
5\end{array}$ \\
\hline ESR & $\begin{array}{l}17.8 \\
5\end{array}$ & $\begin{array}{l}14.1 \\
0\end{array}$ & $\begin{array}{l}7.29 \\
7\end{array}$ & $\begin{array}{l}1.63 \\
2\end{array}$ & $\begin{array}{l}2.29 \\
8\end{array}$ & $\begin{array}{l}0.03 \\
31\end{array}$ & 21 \\
\hline
\end{tabular}

\begin{tabular}{|l|l|l|l|l|l|l|l|}
\hline fasting and & $\begin{array}{l}86.1 \\
0\end{array}$ & $\begin{array}{l}83.3 \\
2\end{array}$ & $\begin{array}{l}28.8 \\
5\end{array}$ & 6.45 & $\begin{array}{l}0.44 \\
94\end{array}$ & $\begin{array}{l}0.65 \\
82\end{array}$ & 3.22 \\
\hline $\begin{array}{l}\text { Postprandi } \\
\text { al }\end{array}$ & $\begin{array}{l}99.7 \\
5\end{array}$ & $\begin{array}{l}100 . \\
55\end{array}$ & $\begin{array}{l}33.0 \\
5\end{array}$ & 7.39 & $\begin{array}{l}0.10 \\
82\end{array}$ & $\begin{array}{l}0.91 \\
49\end{array}$ & $\begin{array}{l}- \\
1.00 \\
2\end{array}$ \\
\hline Lipid profile \\
\hline $\begin{array}{l}\text { Total } \\
\text { cholesterol }\end{array}$ & $\begin{array}{l}184 . \\
9\end{array}$ & $\begin{array}{l}176 . \\
95\end{array}$ & $\begin{array}{l}11.1 \\
9\end{array}$ & 2.50 & $\begin{array}{l}3.17 \\
7\end{array}$ & $\begin{array}{l}0.00 \\
5\end{array}$ & 4.29 \\
\hline $\begin{array}{l}\text { Sr. } \\
\text { triglycerid } \\
\text { es }\end{array}$ & $\begin{array}{l}191 . \\
3\end{array}$ & $\begin{array}{l}187 . \\
35\end{array}$ & $\begin{array}{l}10.5 \\
5\end{array}$ & 2.35 & $\begin{array}{l}1.67 \\
4\end{array}$ & $\begin{array}{l}0.11 \\
04\end{array}$ & 2.06 \\
\hline
\end{tabular}

\section{DisCUSSION}

The term Sciatica refers to pain beginning in the lumbar region and spreading down the back of one lower limb to the ankle or foot. There is usually little weakness or sensory loss but sometimes diminution or loss of the ankle jerk. The recurrence rate of sciatica is a major concern. Advances in science have not yielded substantial treatment option on the pain with the help of analgesics and steroidal therapy. However, its role in treating the disease remains doubtful. As Ayurvedic treatment is believed to manage the root cause of the disease. Therefore, the whole medical fraternities are looking towards this ancient medical science. The present study entitled at the aims to study and to find out the effects of Panchakarma and Anushalya procedures in Sciatica.

\section{Age Wise Distribution}

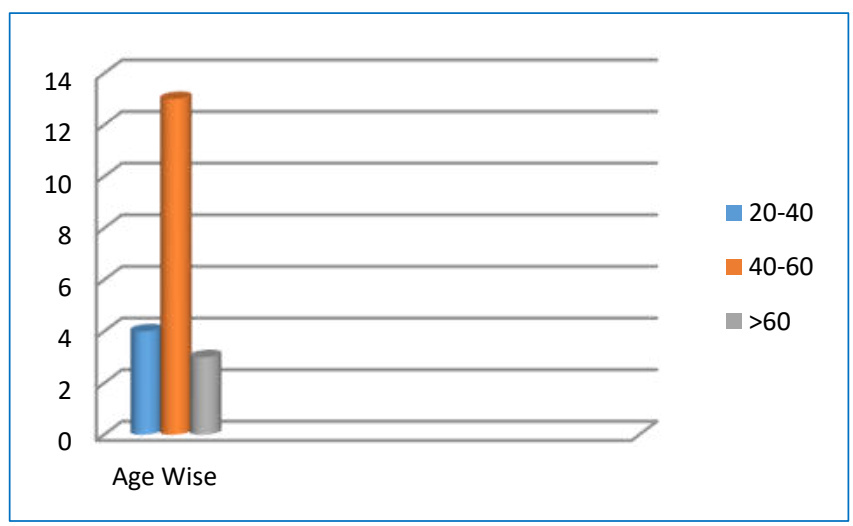

Age distribution was divided into three groups. (32.5\%) patients were belonging to the age group of $20-40$ years. $(60 \%)$ patients were belonging to the age group of $40-60$ years. (7.5\%) were belonging to the 
age group of group 61 onwards. This data suggest that most of these patients fall in the age group of 40-60 years.

\section{Sex Wise Distribution}

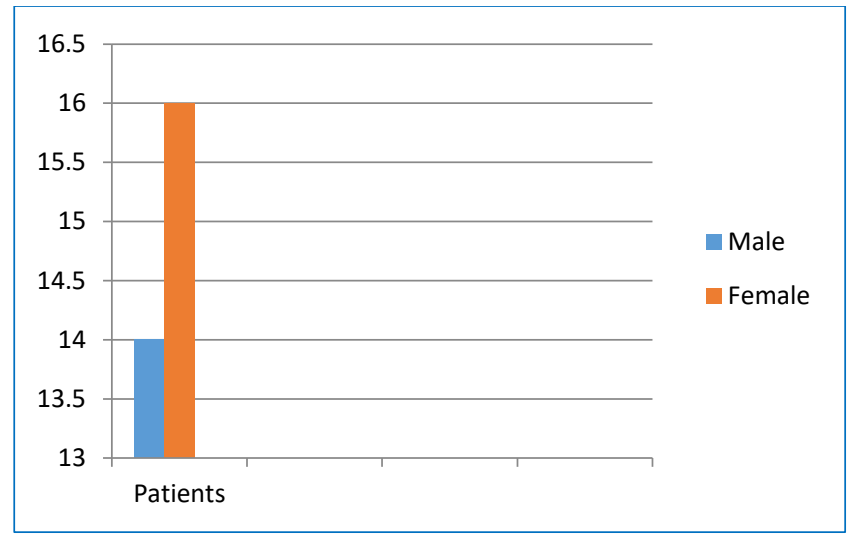

Out of 30 patients there were (45\%) male and (55\%) female.

\section{Chronicity Wise Distribution}

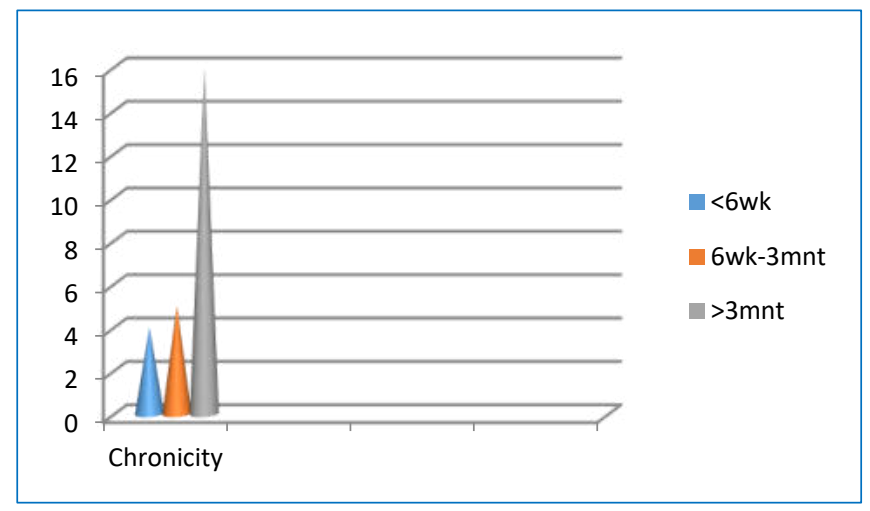

There were (22.5\%) patients of 6 weeks to 3 months chronicity and (77.5\%) patients of chronicity more than 3 months.

\section{Effect in Mean Grade, Scores of Investigations}

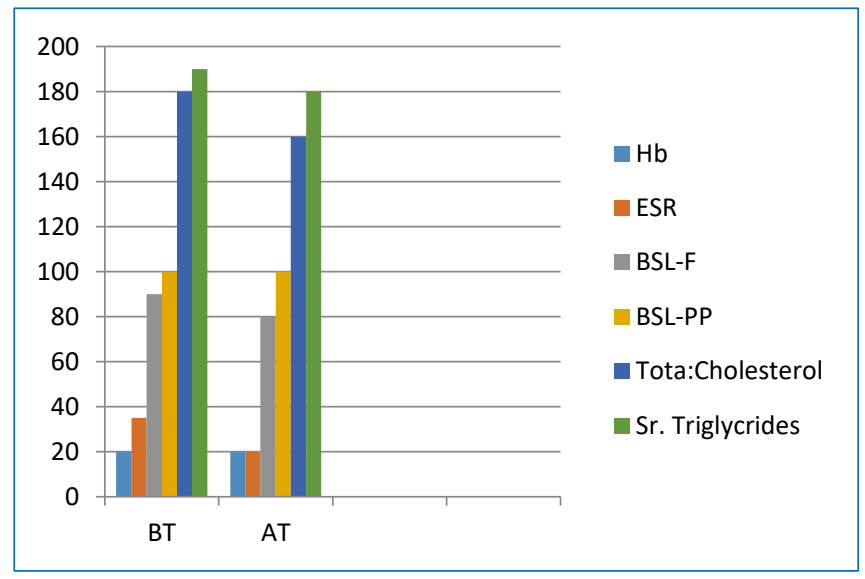

Blood and $\mathrm{X}$ ray- L.S. Investigation in Patients of Sciatica

There were no significant changes seen in patients Sciatica of before and after treatment. Detail values in both groups before and after treatment.

An objective assessment by blood investigation and $\mathrm{X}$ ray - L.S shows.

- No significant change in both the groups.

- No untoward effects have been observed in any of the patients in either treatment group.

\section{CONCLUSION}

The pain relief provided and sustained improvement by Panchatikta Kshira Basti presents a window of opportunity in the clinical management of Sciatica. There were no significant changes seen in blood and radiological investigation in patients of Sciatica before and after treatment.

\section{REFERENCES}

1. Gogate, R.B., 2006: Viddha Aani Agnikarma Chikitsa. 1st Ed. Vaidya Mitra Prakashan, Pune.

2. Chaurasia, B.D., 1995: Human Anatomy. 3rd Ed. C.B.C. Publishers, New Delhi.

3. Mahajan, B.K., 1997: Methods of Biostatistics. 6th Ed. Jaypee Brothers. Medical Publishers P (Ltd), New Delhi, India.

4. Ashish Mhatre1, D.M. Padavi1, D.S. Bhadlikar1 and Mukesh Shukla2, 2015 A Comparative Clinical Studies of Vajigandhadi Taila Basti and Agnikarma in the Management of Sciatica

5. John Walton, 1993: Brain's Diseases of the Nervous System. 10th Ed. Edited by, Oxford University Press.

6. Michael Swash, 2000: Hutchison's Clinical Methods, 12th Ed. W.B. Saunders Company Ltd.

7. Mehta, P.J., 2005: Practical Medicine. 17th Ed. Edited by Mehta, S.P., Joshi, S.R., Mehta, N.P. Published by Shilpa Pradip Mehta, Mumbai, India.

8. Nanda G.C., Padhi M.M., Chopra K.K. Effect of Trayodashang Guggul Along With Abhyanga-Sweda in the Management of Gridhrasi. J.R.A.S. 1998. 14; 116121 
9. Kashinath Shastri and Gorakhnath Chaturvedi, 2001: Charak Samhita of Agnivesha. Revised by Charak and Drudhabala with elaborated 'Vidyotini' Hindi Commentary by, Chaukhamba Bharati Academy, Varanasi, India.
How to cite this article: Dr. Mridula Pathak, Dr. Ashish Mhatre. Clinical efficacy of Panchatikta Kshira Basti in the management of Gridhrasi. J Ayurveda Integr Med Sci 2020;1:45-51.

http://dx.doi.org/10.21760/jaims.5.1.9

Source of Support: Nil, Conflict of Interest: None declared.

Copyright (C) 2020 The Author(s); Published by Maharshi Charaka Ayurveda Organization, Vijayapur (Regd). This is an open-access article distributed under the terms of the Creative Commons Attribution License (http://creativecommons.org/licenses/by/4.0), which permits unrestricted use, distribution, and reproduction in any medium, provided the original work is properly cited. 